\title{
Effects of Electrode Placement and Measurement Time on Electrochemical Signal of Redox Species Flowing through Porous Material
}

\author{
Wataru Iwasaki, ${ }^{1 *}$ Ramachandra Rao Sathuluri, ${ }^{1}$ Ryoji Kurita, ${ }^{1,2}$ \\ Osamu Niwa, ${ }^{1,2,3}$ and Masaya Miyazaki ${ }^{1,4}$ \\ ${ }^{1}$ Advanced Manufacturing Research Institute, National Institute of Advanced Industrial Science and Technology, \\ 807-1 Shuku-machi, Tosu, Saga 841-0052, Japan \\ ${ }^{2}$ Biomedical Research Institute, National Institute of Advanced Industrial Science and Technology, \\ 1-1-1 Higashi, Tsukuba, Ibaraki 305-8566, Japan \\ ${ }^{3}$ Advanced Science Research Laboratory, Saitama Institute of Technology, \\ 1690 Fusaiji, Fukaya, Saitama 369-0293, Japan \\ ${ }^{4}$ Cool Earth Co., Ltd., 8-2 Shinbashi Odori, Kushiro, Hokkaido 100-0005, Japan
}

(Received August 19, 2016; accepted November 25, 2016)

Keywords: electrochemical measurement, immunochromatography, paper-based analytical device, pointof-care testing

We have studied the construction of an electrochemical immunochromatography platform as one of the point-of-care testing devices from both experimental and theoretical aspects. In the electrochemical immunochromatography measurement, the layout of working, reference, and counter electrodes and the flow rate in a porous membrane are important in obtaining a highly sensitive and stable electrochemical signal. In this manuscript, we report the effects of the layout of the three electrodes and the elapsed time after sample injection on electrochemical signals. We found that the working electrode should be placed upstream of the reference electrode because an unfavorable electrochemical signal from a $\mathrm{Ag}^{+}$ion from the reference electrode was observed on the working electrode when it was located downstream of the reference electrode. Furthermore, we found that the electrochemical signal was inversely proportional to the sixth root of time. Experimental and theoretical results are consistent with each other, exhibiting a high correlation coefficient of 0.99 .

\section{Introduction}

Point-of-care testing (POCT) is an easy, fast, and low-cost diagnostic method conducted at the bedside, near the patient, and on-site.(1) The quality of life (QOL) of patients is expected to be improved through the regular monitoring of blood components with low invasive diagnosis by POCT. The portable glucose sensor is one of the representative diagnostic devices for POCT. ${ }^{(2,3)}$ It enables diabetic and prediabetic patients to measure and control their blood glucose concentration by themselves. Immunochromatography is also one of the diagnostic methods for biomarkers such as human chorionic gonadotropin (hCG) in urine for a pregnancy test. It had been applied to various diagnoses because of its simple use and short measurement time of within a few tens

"Corresponding author: e-mail: wataru.iwasaki@aist.go.jp 
of minutes, and it costs less than 10 dollars per measurement. However, it is not quantitative and is difficult to achieve a low detection limit. Martinez et al. introduced a microfluidic paperbased analytical device ( $\mu \mathrm{PAD}$ ) in which paper is used as the substrate for the immunoassay. ${ }^{(4)}$ They demonstrated various biosensing techniques, for example, paper-based enzyme-linked immunosorbent assay (ELISA) and paper-based electrochemical glucose sensing, which can be accomplished with the commercialized glucose sensor. ${ }^{(5,6)} \mu$ PADs have attracted attention not only for human diagnosis but also for use in agricultural fields and environmental measurement because of their very low cost measurement and easy fabrication methods. Therefore, $\mu$ PADs have been studied by several groups in the last decade. There are colorimetric, fluorescent, and electrochemical methods for the quantitative measurement of the amount of biomarkers. In the case of electrochemical methods, the working electrode (WE), counter electrode (CE), and reference electrode (RE) are attached or printed on the paper, and are used to measure the electrochemical species flowing through a paper membrane. ${ }^{(7-9)}$ The placements of WE, CE, and RE are very important for electrochemical measurement. However, the effects of the electrode placement on the detection signal in the paper-based analysis were not investigated. Therefore, we demonstrated the electrochemical measurement of redox species flowing through a porous material using two types of electrochemical detectors that have different placements of each electrode. Furthermore, the flow rate is also important in electrochemical measurement. Therefore, we investigated the relationship between the electrochemical signal and the elapsed time after the sample solution was applied to the porous material.

\section{Materials and Methods}

\subsection{Reagents and apparatus}

The photoresist for the lift-off process, AZ 5214E, and its AZ Remover 700 were purchased from AZ Electronic Materials (Tokyo, Japan). $\mathrm{Ag} / \mathrm{AgCl}$ ink was purchased from BAS Inc. MA10 (Mikasa Co., Ltd, Tokyo, Japan) was used for photolithography. Electron beam deposition equipment (Cosmo-system, Fukuoka, Japan) was used for the deposition of a gold thin film. Three nitrocellulose membranes (HF75, HF120, HF180; Merck Millipore Co. Ltd., Massachusetts, USA) with different flow rates were used to investigate the effect of the flow rate in this study. The flow durations with respect to a distance of $40 \mathrm{~mm}$ were 75,120 , and $180 \mathrm{~s}$ for HF75, HF120, and HF180 membranes, respectively. We used $p$-aminophenol ( $p$ AP, 015-02692, Wako Pure Chemical Industries, Ltd., Osaka, Japan) as a representative electrochemical species for electrochemical measurement because $p \mathrm{AP}$ has been detected as a product of the labeled enzyme used in electrochemical enzyme immunoassay. A numerical control micromilling machine (Micro MC2, PMT corp., Fukuoka, Japan) was used for the patterning of a polymethylmethacrylate (PMMA) plate.

\subsection{Electrochemical immunochromatography platform}

The electrochemical detector was fabricated by standard photolithography and the liftoff process..$^{(7)}$ First, a photoresist was spin-coated on a silicon wafer with a $1-\mu \mathrm{m}$-thick thermal oxide silicon film and patterned by photolithography with the electrode pattern. The widths of the WE and RE were $1 \mathrm{~mm}$ and that of CE was $2 \mathrm{~mm}$. The electrodes were placed at $0.5 \mathrm{~mm}$ intervals. Secondly, 
a 200-nm-thick gold thin film was deposited on the surface of the silicon wafer that was deposited with a 5-nm-thick chromium thin film that had been formed by electron beam deposition. Then, the gold electrode was patterned by removing the remaining photoresist on the silicon wafer (liftoff process). Finally, $\mathrm{Ag} / \mathrm{AgCl}$ ink was painted on one gold electrode as the $\mathrm{RE}$ and dried at $70{ }^{\circ} \mathrm{C}$ for $2 \mathrm{~h}$. The total size of the electrochemical detector was $10 \mathrm{~mm}$ wide and $20 \mathrm{~mm}$ long. We fabricated two types of detector with different placements of the WE and RE to investigate the effect of the arrangement of electrodes on the signal.

Figure 1(a) shows the Type A detector in which the WE is located upstream of the RE. On the other hand, Type B has the WE downstream of the RE. The electrochemical detector was assembled with the electrochemical measurement platform, as shown in Fig. 1(b). The platform consists of a nitrocellulose membrane, absorbent pad, electrochemical detector, and two PMMA plates. The nitrocellulose membrane $5 \mathrm{~mm}$ wide and $50 \mathrm{~mm}$ long was attached to the electrochemical detector and the absorbent pad, sandwiched between the two PMMA plates. The two PMMA plates were held with screws. The top PMMA plate has an inlet upstream of the nitrocellulose membrane. The other side of the nitrocellulose membrane is connected to the absorbent pad. The electrochemical detector was positioned at the center of the membrane.

\subsection{Electrochemical measurement}

We conducted two experiments. We investigated the effect of the arrangement of electrodes using two types of electrochemical detectors (Types A and B) in the first experiment. First, $10 \mu \mathrm{g} /$ $\mathrm{ml} p \mathrm{AP}$ in $20 \mathrm{mM}$ tris buffered saline with $0.05 \%$ Tween 20 (TBST) was applied in the inlet of the platform. Then, we conducted cyclic voltammetry $(\mathrm{CV})$ with the range of potential from -0.3 to $0.3 \mathrm{~V}$ vs $\mathrm{Ag} / \mathrm{AgCl}$ at the scan rate of $50 \mathrm{mV} / \mathrm{s}$ after the wetting front reached the absorbent pad. $\mathrm{CV}$ was conducted five times at 1-min intervals for the electrochemical detector. HF120 was used in the first experiment.

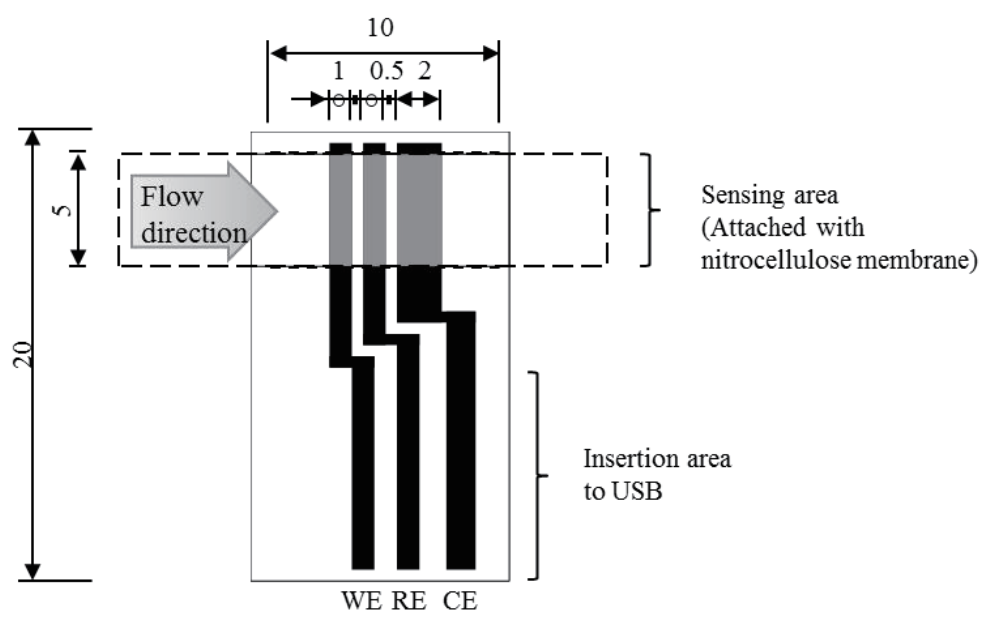

(a)

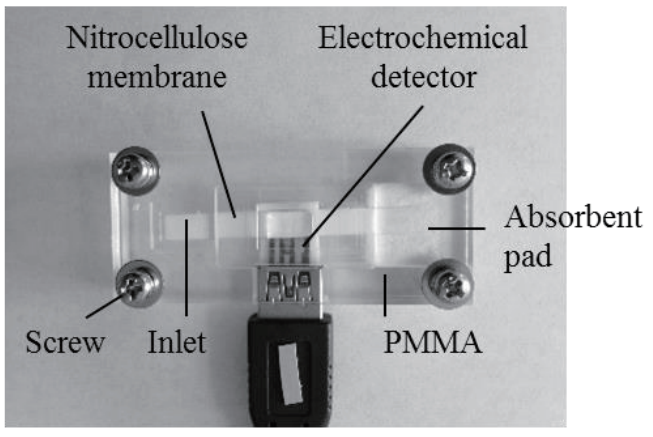

(b)

Fig. 1. Schematic of electrochemical detector (a) and photograph of electrochemical immunochromatography platform (b). WE, RE, and CE represent working, reference, and counter electrodes, respectively. The arrangement of the electrodes of this figure is Type A. In Type B, the positions of WE and RE are switched. 
In the second experiment, we investigated the effect of the elapsed time after applying the sample solution on the measured signal. In this experiment, the electrochemical detector Type A was used, and $10 \mu \mathrm{g} / \mathrm{ml} p$ AP in $20 \mathrm{mM}$ TBST was used as the sample solution, as in the first experiment. We started chronoamperometry with an applied electrical potential of $0.2 \mathrm{~V} \mathrm{vs} \mathrm{Ag} /$ $\mathrm{AgCl}$ after the sample solution was injected into the platform. Three membranes, namely, HF75, HF120, and HF180, were used for the second experiment to investigate the differences in the flow rate.

\section{Results and Discussion}

\subsection{Effect of the position of RE on electrochemical signal}

Figure 2 shows a cyclic voltammogram measured using the electrochemical detector with the WE located upstream (Type A) and downstream (Type B) of the RE. The dashed line shows the first measurement that was conducted just after the wetting front passed the $\mathrm{CE}$ and the solid line represents the sixth measurement that was conducted $5 \mathrm{~min}$ after the first measurement. Each voltammogram shows obvious redox peak currents of $p \mathrm{AP}$ at $0.03 \mathrm{~V}$ vs $\mathrm{Ag} / \mathrm{AgCl}$ and $-0.03 \mathrm{~V}$ vs $\mathrm{Ag} / \mathrm{AgCl}$. In the case of Type $\mathrm{B}$, another reduction peak current was observed at around -0.04 $\mathrm{V}$ vs $\mathrm{Ag} / \mathrm{AgCl}$ after $5 \mathrm{~min}$. On the other hand, there is no additional reduction signal for Type A. It is considered that $\mathrm{Ag}$ of the $\mathrm{RE}$ was dissolved in solution and $\mathrm{Ag}^{+}$ions flowed through the membrane. Then, $\mathrm{Ag}^{+}$ions were detected at the WE in the case of Type $\mathrm{B}$, because the WE was located downstream of the RE. In contrast, $\mathrm{Ag}^{+}$ions did not affect the measured redox signal of $p \mathrm{AP}$ because the WE was located upstream of the RE in Type A. The reduction signal derived from $\mathrm{Ag}^{+}$can be distinguished from the $p \mathrm{AP}$ signal by the potential sweep technique including a CV. However, the electrochemical detector in the flow systems including our device can be operated at

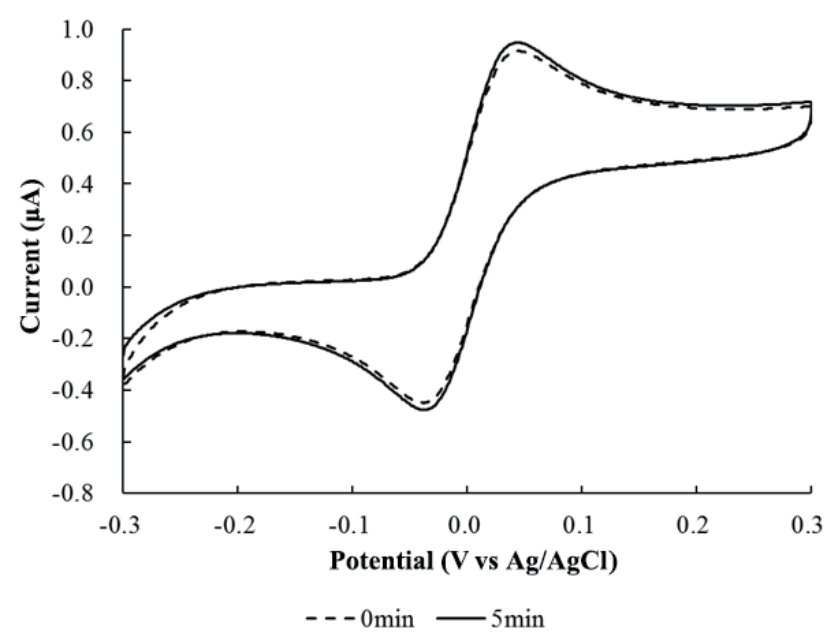

(a)

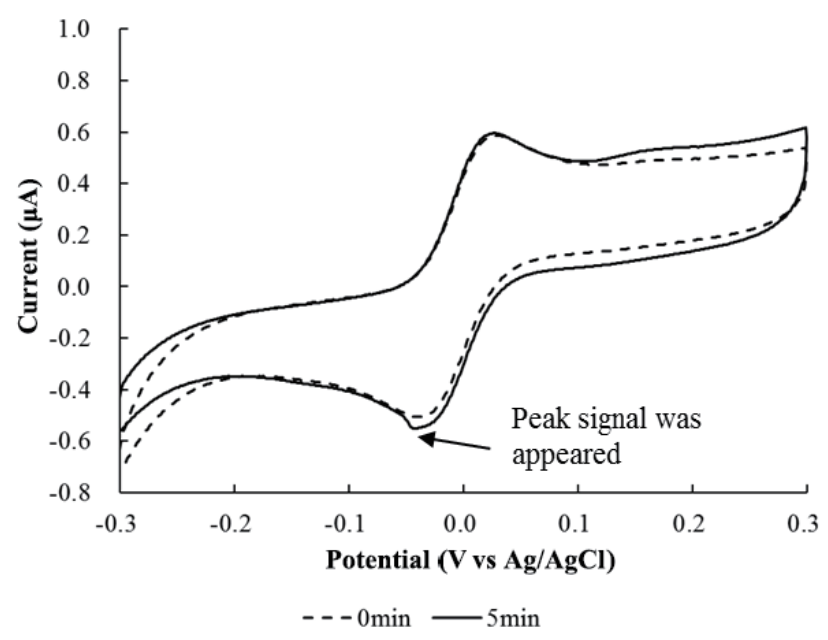

(b)

Fig. 2. Cyclic voltammogram of $10 \mu \mathrm{g} / \mathrm{ml} p \mathrm{AP}$ in $20 \mathrm{mM}$ TBST measured with Type A (a) and Type B (b) electrochemical detectors at the scan rate of $50 \mathrm{mV} / \mathrm{s}$. Type A has the WE located upstream of the RE and Type B has the WE located downstream of the RE. 
constant potential owing to its simplicity. At the constant potential mode, we cannot remove the effect of $\mathrm{Ag}^{+}$ions, which interfere with quantitative detection. Therefore, it is important to position the RE downstream of the WE.

\subsection{Effect of time on electrochemical signal}

Figure 3 shows the oxidation current of $10 \mu \mathrm{g} / \mathrm{ml} p$ AP in $20 \mathrm{mM}$ TBST flowing through nitrocellulose membranes with different flow rates measured by chronoamperometry (solid line). The oxidation current was not measured until the wetting front reached the electrodes because the oxidation current can be detected when the surfaces of the three electrodes were wetted. In the case of the HF120 membrane, two peak signals were observed at 38 and $44 \mathrm{~s}$ when the wetting front reached the WE and RE, respectively. After that, a continuous stable oxidation current was measured. However, the oxidation current had gradually decreased until the wetting front reached the absorbent pad. This is because flow rate decreases with increasing distance between the wetting front and the inlet. If the nitrocellulose membrane is regarded as a microchannel, oxidation current $(i)$ with a planar electrode in parallel flow to the electrode in the channel is defined as

$$
i=1.47 n F C(D A / b)^{2 / 3} v^{1 / 3},
$$

with the valence $(n)$, Faraday constant $(F)$, concentration $(C)$, diffusion coefficient $(D)$, electrode area $(A)$, channel height $(b)$, and flow rate $(v) .^{(10)}$ The relationship between the flowing distance of a liquid flowing through a porous material $(L)$ and time $(t)$ is defined using the Lucas-Washburn equation as

$$
L=\sqrt{\frac{k \gamma t \cos \theta}{\varphi \mu d}},
$$

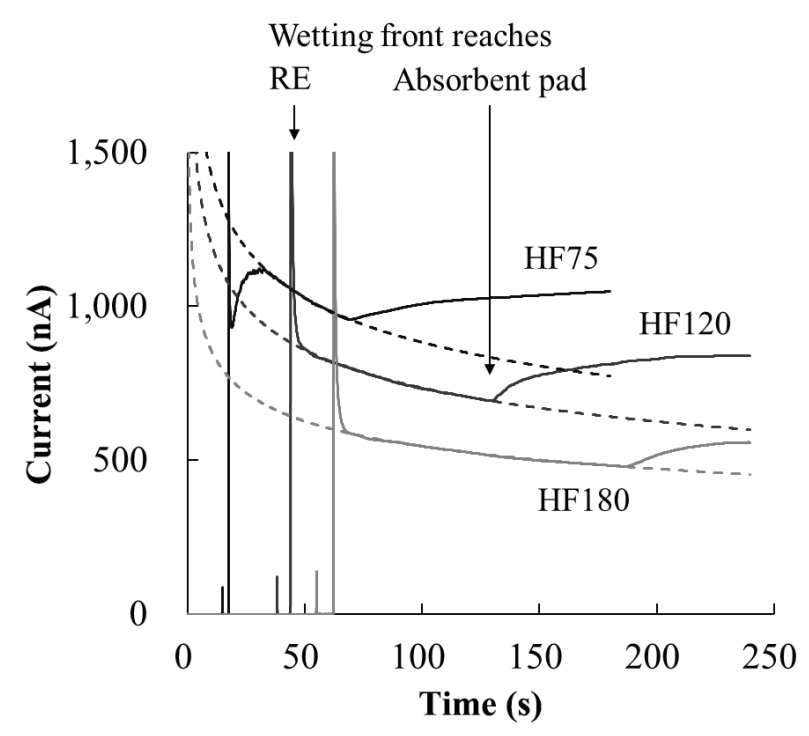

Fig. 3. Oxidation current of $10 \mu \mathrm{g} / \mathrm{ml} p$ AP in $20 \mathrm{mM}$ TBST flowing through nitrocellulose membranes with different flow rates. The solid and dashed lines represent measured and calculated signals, respectively. 
with the surface tension $(\gamma)$ and viscosity $(\mu)$ of the flowing fluid, the liquid/solid contact angle $(\theta)$, permeability $(k)$, porosity $(\varphi)$, and the mean diameter of the pores of the membrane $(d)$. The flow rate is inversely proportional to the square root of the time.

$$
v \propto t^{-1 / 2}
$$

That is, the oxidation current is inversely proportional to the sixth root of time from Eqs. (1) and (3):

$$
i=k t^{-1 / 6}+a,
$$

where $k$ and $a$ are arbitrary constants. The dashed lines in Fig. 3 show the calculated signal that is inversely proportional to the sixth root of time. The arbitrary constants were chosen by curve fitting every measurement. They overlapped with the measured signals. The contribution ratios between the measured and calculated signals were more than 0.99 in each flow rate. On the other hand, the electrochemical signal became constant after the wetting front reached the absorbent pad, because the flow rate became constant. Thus, we must consider where the wetting front is to enable quantitative measurement. As a result of the above study, we concluded that the measurement should be conducted after the wetting front reaches the absorbent pad in order to obtain a stable result of the electrochemical immunoassay.

\section{Conclusions}

We studied the construction of an electrochemical immunochromatography platform as one of the point-of-care testing devices from both experimental and theoretical aspects. The platform was easily used merely by inserting a USB connector that is connected to a potentiostat. Furthermore, a sufficient contact between the electrode and the membrane was established for stable measurement. We investigated the effect of the layout of the electrodes of the electrochemical detector on the electrochemical measurement. We found that an undesirable reduction current derived from $\mathrm{Ag}^{+}$ ions from the RE was observed when the RE was located upstream of the WE. On the other hand, when the RE was located downstream of the WE, no undesirable signal was observed. This signal derived from $\mathrm{Ag}^{+}$interferes with the quantitative detection because our system adopts a constant potential and cannot remove the effect of the $\mathrm{Ag}^{+}$signal. Furthermore, we investigated the effect of the flow rate of the redox species. We found that the oxidation current of $p \mathrm{AP}$ decreased inversely proportional to the sixth root of time until the wetting front reached the absorbent pad regardless of the flow rate and became stable after the wetting front reached the absorbent pad. We concluded that we should avoid these effects to successfully accomplish quantitative results with immunochromatography based on the electrochemical method by using an electrochemical detector that has the RE located downstream of the WE and conducting the measurement after the wetting front reaches the absorbent pad.

\section{Acknowledgements}

This work was partly supported by the Council for Science, Technology and Innovation (CSTI), Cross-ministerial Strategic Innovation Promotion Program (SIP), "Technologies for creating next-generation agriculture, forestry and fisheries" (funding agency: Bio-oriented Technology 
Research Advancement Institution, NARO) and by the Japan Society for the Promotion of Science KAKENHI Grant number 16K06036.

\section{References}

1 A. Warsinke: Anal. Bioanal. Chem. 393 (2009) 1393.

2 E. H. Yoo and S. Y. Lee: Sensors 10 (2010) 4558.

3 D. R. Matthews, E. Bown, A. Watson, R. R. Holman, J. Steemson, S. Hughes, and D. Scott: Lancet 1 (1987) 778.

4 A. W. Martinez, S. T. Phillips, M. J. Butte, and G. M. Whitesides: Angew. Chem. Int. Ed. 46 (2007) 1318.

5 C. M. Cheng, A. W. Martinez, J. L. Gong, C. R. Mace, S. T. Phillips, E. Carrilho, K. A. Mirica, and G. M. Whitesides: Angew. Chem. Int. Ed. 49 (2010) 4771.

6 Z. H. Nie, F. Deiss, X. Y. Liu, O. Akbulut, and G. M. Whitesides: Lab Chip 10 (2010) 3163.

7 W. Iwasaki, R. R. Sathuluri, O. Niwa, and M. Miyazaki: Anal. Sci. 31 (2015) 729.

8 T. Yasukawa, Y. Kiba, and F. Mizutani: Anal. Sci. 31 (2015) 583.

9 M. R. Akanda, H. A. Joung, V. Tamilavan, S. Park, S. Kim, M. H. Hyun, M. G. Kim, and H. Yang: Analyst 139 (2014) 1420.

10 A. J. Bard and L. R. Faulkner: Electrochemical Methods: Fundamentals and Applications (Wiley, New York, 2001) 2nd ed. 